\title{
Novel Fractional order Integrators Using Traditional Rules Interpolation
}

\author{
Chafia MEKHNACHE ${ }^{(1)}$, Nawel BALASKA ${ }^{(2)}$, Nedjma DETOUCHE ${ }^{(3)}$ \\ (1) (2) (3) Electrical engineering departement, ENSET Cité des frères boucetta azzaba \\ Skikda,Algérie \\ ${ }^{(1)} \underline{\mathrm{cm} o k h n a c h e @ y a h o o . f r,}{ }^{(2)}$ nabalaska@yahoo.fr, ${ }^{(3)}$ ndetouche@yahoo.fr
}

\begin{abstract}
This work proposes a new IIR integer order digital, so a new IIR fractional order integrators. The followed method includes two stages. First, the integer order integrator is obtained by interpolating well-known integration rulesnamely, Euler, bilinear and Simpsonas a weighted sum. Second the initial value theorem for the selection of initial value of the impulse response is used. Lastlythe Steiglitz-Mc-bride signal modeling technique is applied to find the parameters of rational approximation models. Numerical examples are presented to illustrate the performance of the proposed integrator. It was found that the Euler-bilinear-Simpson integrator yields high accuracy than the existing integrators.
\end{abstract}

Keywords: Digital integrators, Fractional order integrators, Impulse invariant method, Euler-bilinear-Simpson integrator, Steiglitz-Mc-bride

\section{INTRODUCTION}

The device that can be used to calculate the times integral of a given signal is named integrator. It is very essential in various applications such as control systems [1], [2], biomedical engineering [3], [4], radar and electronic circuits [5], [6], and image processing [7], [8] ...etc.

Digital integrator can be either FIR [9], [10], [11], [12] or an IIR [13], [14], [15], [16]. Our interested type of integrators here is IIR ones. They can be obtained directly from the well-known rectangular trapezoidal methods of numerical integration [9], [10], [17], [18]. First and second order integrators were constructed by Al-Alaoui using the combination between the rules: rectangular, and trapezoidal [13], [14], or between rectangular, and Simpson [14].

Fractional order digital differentiator is an extended version of integer order differentiator which offers better practical results in some applications such as transmission line model [19], heat conduction model [20],[21], ... etc. For this raison, various design methods of the fractional operator $D^{v}$ ( $v$ is a real number) in continuous and discrete time domains have been explored by many researchers. They are power series expansion (PSE) [22], continuous fractional expansion (CFE) [23], series expansion design using logarithm [24] and impulse invariance/signal modeling method, [25], [26]...etc. This latter is used here to avoid the complexity when a larger number of the impulse response samples are required or higher order $\mathrm{s}$ to $\mathrm{z}$ transforms are used.

The method of designing digital integrators or differentiators by interpolating two rules already exist in literature [13], [14], [27], [28]. However, we focus our contribution here on the rules which are not used yet. Firstly, we combine the Euler [13], bilinear [13] and the Simpson [18] rules with a parameter "a". The obtained integer order IIR integrator is used in the design of fractional order IIR integrator, so we call the initial value theorem for the selection of initial value of the impulse response, finally we apply Steiglitz-Mc-bride signal modeling technique to find the parameters of rational approximation models. Thereafter, the fractional integral of some deterministic signals, such as the sawtooth signal can be computed.

The paper is divided into five sections including the introduction and conclusion. Section 2 explains the mixed method between the Euler, bilinear and Simpson rules. The impulse invariance/signal modeling method is reviewed briefly in section 3. Numerical examples are included in the section 4 to illustrate the effectiveness of the proposed integrator.

\section{THE PROPOSED METHOD}

Ideal integrator 
The ideal first-order digital integrator has frequencyresponse:

$$
D(\omega)=\frac{1}{j \omega} \quad|\omega| \leq \pi
$$

and repeats with period $2 \pi$.

The ideal integrator has a constant phase response of $-90^{\circ}$. It is discontinuous at half the sampling frequency and approximation of such phase is difficult. Thus, the ideal integrator used here as a reference is supposed to be: $\quad H_{d}(\omega)=e^{j \omega T / 2} /(j \omega)[29]$ to overcome this problem of phase discontinuity [30], noting that the magnitude response is not affected by using this integrator.

Here, we reminder that the new digital integrator is proposed such that its frequency response fits $H_{d}(\omega)$ as well as possible. The filter coefficients are obtained by combining Euler, bilinear and Simpson integrators as a weighted sum. The interpolation of the chosen rules is given in the following subsections.

\section{Euler-bilinear-Simpson integrator}

The transfer functions of integrators to be mixed which are: Euler, bilinear and Simpson integrators are given respectively by:

$$
\begin{array}{r}
H_{E u}(z)=T \frac{z}{z-1}(2) \\
H_{b i}(z)=\frac{T}{2} \frac{z+1}{z-1}(3) \\
H_{S}(z)=T \frac{2 z}{\left(z^{2}-1\right)}(4)
\end{array}
$$

with $T$ representing the samplingperiod.

The interpolation takes the form:

$$
H_{\text {Eubis }}(z)=a H_{b i}(z)+(1-2 a) H_{S}(z)+a H_{E u}(z)
$$

wherea is a real valued parameter defined on the closed interval [ $\left[\begin{array}{ll}0 & 1\end{array}\right]$.

Substituting (2), (3) and (4) in equation (5), we obtain:

$$
\begin{gathered}
H_{\text {Eubis }}(z)=a \frac{T}{2} \frac{z+1}{z}+(1-2 a) T \frac{2 z}{\left(z^{2}-1\right)}+a T \frac{z}{z-1} \\
H_{\text {Eubis }}(z)=\frac{T\left(3 a z^{2}+4(1-a) z+a\right)}{2\left(z^{2}-1\right)}(6)
\end{gathered}
$$

The magnitude responses of the ideal, Euler, bilinear and Simpson the mixed integrators obtained by substituting $z=e^{j \omega T}$ in the corresponding transfer functions are depicted in Fig. 1 for $a=0.4$.

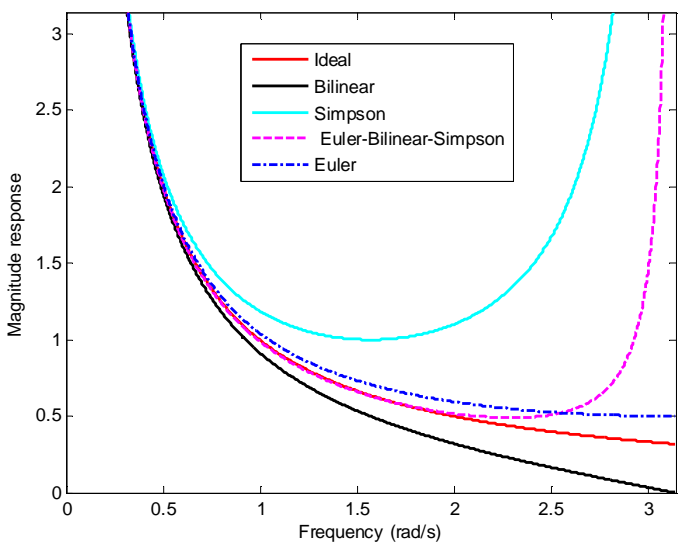

Fig. 1Magnitude responses of ideal, and approximated integrators.

This figure shows clearly that the magnitude response of the obtained integrator match more closely to the ideal response in a certain range, especially at low and in midband frequency. The magnitude responses of Euler, bilinear and Simpson integrators yield better accuracy, only at low frequency.

The phase response of Euler, bilinear, Simpson and hybrid Euler bilinear-Simpson integrators together with that of the ideal one is shown in Fig. 2.

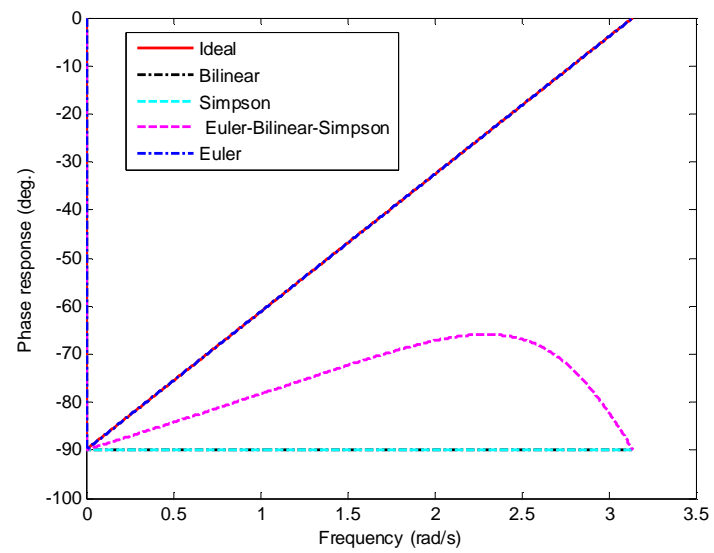

Fig. 2. Phase responses of ideal, and approximated integrators.

Regarding to the phase responses, it can be noticed that the Euler phase integrator is linear, and that of the mixed integrator is almost linear.

The new hybrid integrator introduced here is exploited to compute the fractional order aintegral of deterministic signals in the next section. For this, a review of the impulse 
invariance method is presented in the next section.

\section{DESIGN OFRECURSIVE FRACTIONAL ORDER INTEGRATOR}

The method used here to design recursive fractional order integrator is described in [25]. It is based on the impulse invariance methodto avoid the complexity when a larger number of the impulse response samples are required or higher order $s$ to $z$ transforms are used. The two main steps of this method are as followed:

1. Choose the integrator obtained in the previous section to select the initial value of the impulse response:

$$
h_{\alpha}(0)=\lim _{z \rightarrow \infty} H_{\alpha}(z)
$$

where $\mathrm{H}_{\alpha}(\mathrm{z})$ is the transfer function of discrete time fractional order integrator which is given by:

$$
H_{\alpha}(s)=\frac{1}{s^{\alpha}}
$$

The numerical integration formulas such as Euler $\quad 1 / s \approx T z /(z-1)$, Tustin $1 / s \approx T(z+1) /(2(z-1)), \quad$ Al-Alaoui $1 / s \approx 7 T(z+1 / 7) /(8(z-1))$, was used in references [13], [14]. In this paper, the derived integrator given in eq. (6) is used instead in order to improve the approximation accuracy of the existing proposed integrators.

2. Apply the Steiglitz-Mc-bride signal modeling technique [26] to find the coefficients $a_{\mathrm{i}}$ and bi of the rational transfer function.

$$
H(z)=\frac{\sum_{i=0}^{q} b_{i} z^{i}}{1+\sum_{i=1}^{p} a_{i} z^{i}}
$$

Such that this transfer function $H(z)$ best approximates $H_{\alpha}(z)$ in some sense.

\section{RESULTS AND DISCUSSIONS}

In this section the above procedure was used to compute the fractional integral of sawtooth signal. The following parametersare considered: fractional order $\alpha(0<\alpha<1)$, sampling period $T$, degrees of the approximating rational transfer function $\mathrm{p}, \mathrm{q}$, and first $\mathrm{L}$ samples of discrete impulse response $\quad h_{\alpha}(n)=T^{n} n^{\alpha}{ }^{1} / \Gamma(\alpha) \quad n=0,1,2, \ldots$

[18]. An example is given, it concern sawtooth signal.

\section{Example}

\section{Choosing the parameter a}

In this example, we will compute the fractional integral of 0.4 order of the sawtoothsignal.Its fractional integral of order $\alpha$ is given by [31]:

$$
\left.x(t)=\frac{t^{1+\alpha}}{\Gamma(2+\alpha)}+2 \sum_{k=1}^{N-1}-1\right) u(t-2 k+1) \frac{(t-2 k+1)^{p+\alpha}}{\Gamma(2+\alpha)}(10)
$$

The parameters used here are: $T=0.05, p=5$ $q=5, L=1000$.

In order to investigate the effect of parameter " $a$ " on the design results, we calculate fractional integral of the sawtooth signal, the order $\alpha=0.4$ and the parameter $a=0.2$ to 0.7 with 0.1 increments.

The first sample of discrete impulse response $h_{\alpha}(n)$ is calculated using eq. (7). It is given by:

$$
h_{\alpha}(0)=\left(\frac{3 T a}{2}\right)^{\alpha}
$$

The results are set in table 1.

Table 1. Values of the first sample of the discrete impulse response

\begin{tabular}{|l|l|}
\hline $\begin{array}{l}\text { Integrator Euler- } \\
\text { Bilinear-Simpson } \\
\text { different values of } \\
\text { parametera }\end{array}$ & $\begin{array}{l}\text { First Value of } \\
h_{\alpha}(n): h_{\alpha}(0)\end{array}$ \\
\hline$a=0.2$ & 0.0979148362360976 \\
\hline$a=0.3$ & 0.1151555848912132 \\
\hline$a=0.4$ & 0.1291994009955633 \\
\hline$a=0.5$ & 0.1412617250247383 \\
\hline$a=0.6$ & 0.1519487052336354 \\
\hline$a=0.7$ & 0.1616127869713694 \\
\hline
\end{tabular}

Once $h_{\alpha}(0)$ is calculated, it is easy to calculate the other values of the discrete impulse response $h_{\alpha}(n)$ (using $\left.h_{\alpha}(n)=T^{n} n^{\alpha} 1 / \Gamma(\alpha) \quad n=1,2, \ldots\right)$. Then the coefficients of the rational transfer function $\mathrm{H}(\mathrm{z}) \quad a_{i}$ and $b_{i}$ are obtained using the technique of "Steiglitz-McBride". Finally, these coefficients are employed to find the fractional integration of our test signal.

Figure 3 shows the result of the fractional integration of the sawtooth signal. 


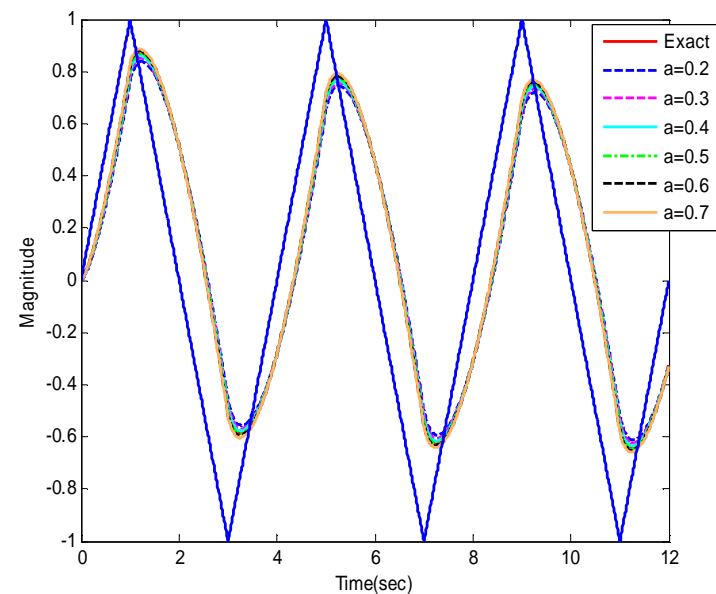

Fig. 3. Fractional integral of the sawtoothsignal: exact and using Euler-Bilinear-Simpson for $\mathrm{a}=0.2$ to $\mathrm{a}=0.7$

It can be seen from Fig. 3, which concern fractional integrals of the sawtooth signal that the all amplitudes match more closely to the exact one. However these amplitudes are superposed, in order to differentiate between these amplitudes, the curves of error of amplitude $E=\left|y_{\text {exact }}-y\right|$ are depicted in Fig. 4.

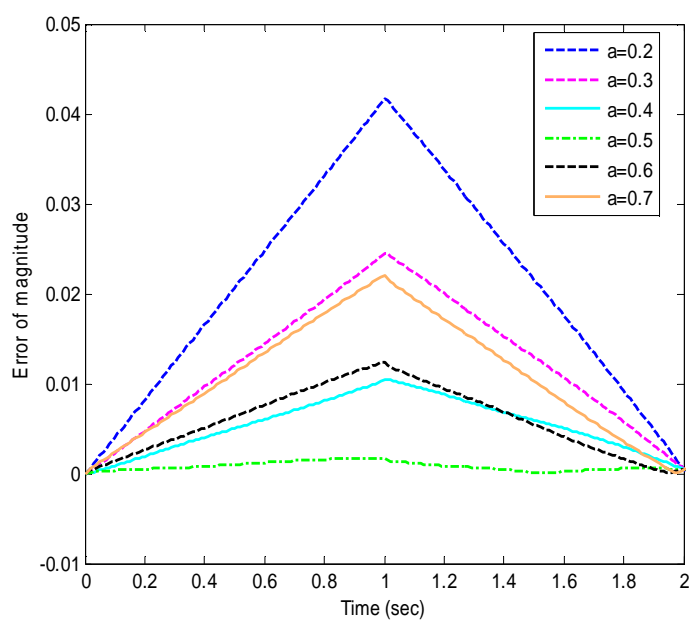

Fig. 4 Error of amplitude

It can be seen from this figure that the proposed fractional integrator based on interpolation between Euler, bilinear and Simpson integration rules with order $\alpha=0.4$ in the case of the parameter $a=0.5 y i e l d s$ highperformance than the other integrators.

The coefficients of the rational transfer function $H(z) a_{i}$ and $b_{i}$ are summarized in Table 2.
Table 2.Coefficients $a_{i}$ and $b_{i}$

\begin{tabular}{|l|l|l|}
\hline & $a_{i}$ & $b_{i}$ \\
\hline \hline & & \\
Mixed & 1.000000000 & 0.1412617295725612 \\
integrator: & -4.091895344580705 & -0.5066208568946221 \\
Euler- & 6.541546199356355 & 0.6792588899405360 \\
Bilinear- & -5.066192881895555 & 0.4057448065868380 \\
Simpson & 1.875380356074513 & 0.09396133188813621 \\
for $a=0.5$ & -0.2588382923164264 & -0.00211613289556519 \\
& & \\
\hline
\end{tabular}

Comparison of Euler-bilinear-Simpson integrator ( $a=0.5)$ with the existing integrators

We want to calculate the integral of fractional order aof sawtooth signal. The parameters used in this example are as follows: $\alpha=0.4, T$ $=0.05, p=5, q=5, L=1000$. The proposed integrator used here is the Euler-bilinearSimpson for $a=0.5$, and the comparison of results is made with the integrators: Al-Alaoui, Euler, bilinear, D. K. Upaddhyay.

The transfer function of D. K. Upaddhyay integrator is given by:

$H_{U P}(z)=\frac{T(z+0.8239)(z+0.295)(z \quad 0.1014)(z+0.0365)}{1.1633(z \quad 1)(z+0.182)(z+0.782)\left(\begin{array}{ll}z & 0.1019\end{array}\right)}$

The values of the first sample of the discrete impulse response are reported in Table 3.

Table 3.values of the first sample of the discrete impulse response

\begin{tabular}{|l|l|}
\hline Integrator & First Value of $h_{\alpha}(n): h_{\alpha}(0)$ \\
\hline \hline Al-Alaoui & {$\left[\frac{7 T}{8}\right]^{\alpha}=0.28601658896468$} \\
\hline Euler & $T^{\alpha}=0.30170881682725$ \\
\hline Tustin & {$\left[\frac{T}{2}\right]^{\alpha}=0.22865252596366$} \\
\hline D.K.Upaddhyay & {$\left[\frac{\mathrm{T}}{1.1633}\right]^{\alpha}=0.2839954031743233$} \\
\hline $\begin{array}{l}\text { Euler-Bilinear- } \\
\text { Simpson }\end{array}$ & {$\left[\frac{1.5 \mathrm{~T}}{2}\right]^{\alpha}=0.2689134392331425$} \\
\hline
\end{tabular}

Fractional integrals of the sawtooth signal are shown in Fig. 5. 


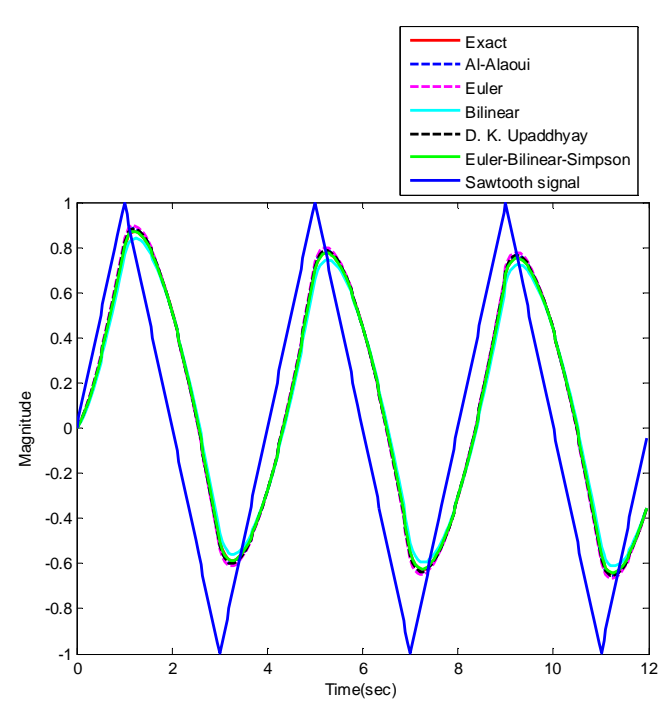

Fig. 5. Fractional integral of sawtooth signal

In order to differentiate all these curves, we depict the errors of each magnitude in Fig. 6.

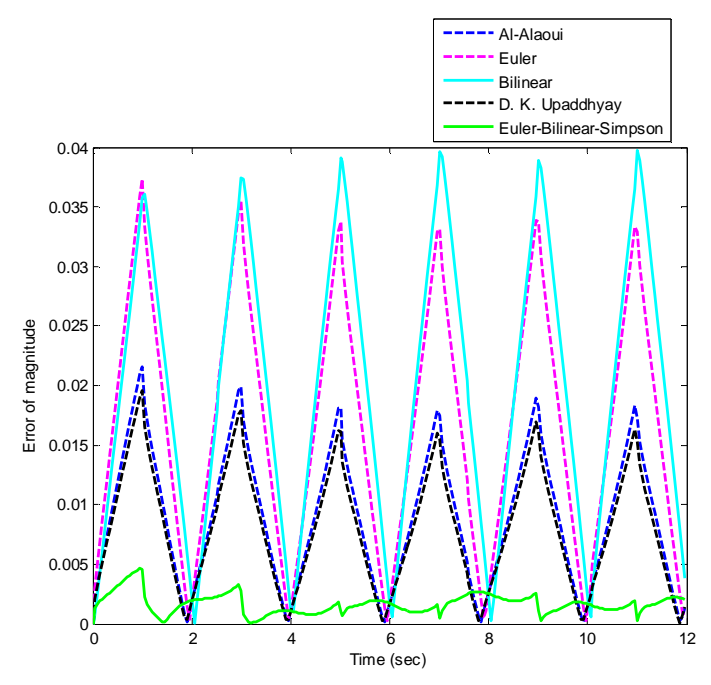

Fig. 6. Error of magnitude

As shown in Fig. 6, there exist a close resemblance between the exact fractional integral of sawtooth signal and calculated fractional integral with the proposed method. However, the Euler, bilinear, D. K. Upaddhyay and Al-Alaoui integrators perform least than theEuler-Bilinear-Simpson integrator.

To check the stability of the obtained fractional integrators, we plot the poles and zeros of each integrator in Fig. 7. 

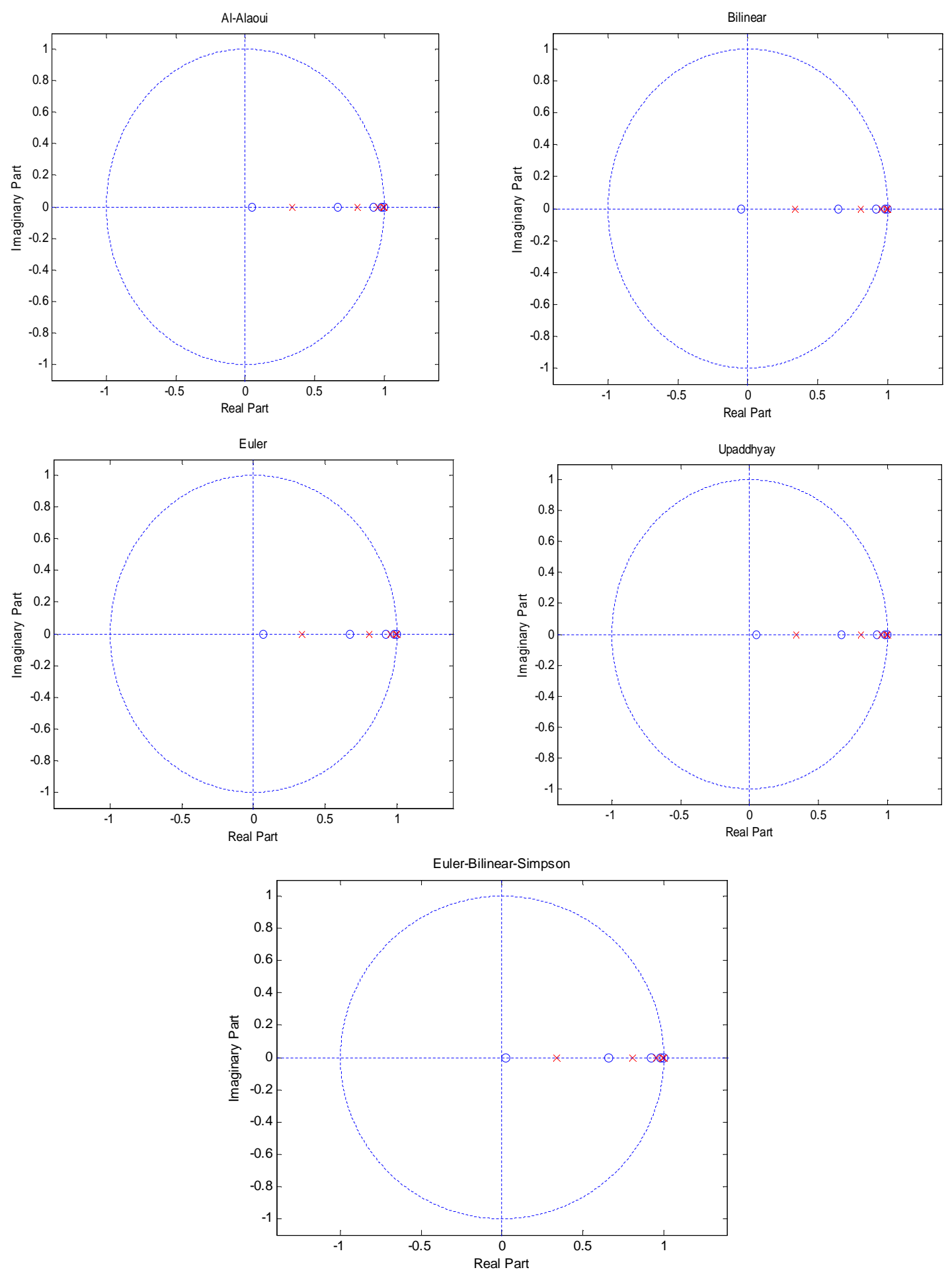

Fig. 7.Pole- zero position of the obtained integrators

As it is known, the filter is stable when all of the poles are inside the unit circle. From the above figure, it's easy to tell that the filter is stable.

\section{CONCLUSION}

This paper is dedicated to the design of integer order IIR digital integrator for the designing of fractional order integrator. The 
main idea was to combine three rules: Euler, Bilinear, and Simpson integrators with a parameter defined between 0 and 1 . Then, the second objective in this work was to employ the obtained integrator for selecting the first sample of the impulse response, then applying signal modeling technique to find the parameters of rational approximation models. TheMATLAB simulation results validate thatthe effective performance of the proposed method over the existing methods which are Euler, Al-Alaoui, bilinear and D.K. Upaddhyay. Other combinations and their applications in edge detection and ECG preprocessing will be investigated in the future.

\section{References}

[1] C. Candan, "Digital Wideband Integrators with Matching Phase and Arbitrarily Accurate Magnitude Response," IEEE Trans. Circuits and Systems II, Vol. 58, N. 9, pp. 610-614, September. 2011.

[2] G.F. Franklin, J.D. Powell, M.L. Workman. "Digital control of dynamics systems", 2nd ed. Addison-Wesley, Reading. 1990.

[3] B.T.Krishna, "Studies on fractional order differentiators and integrators: A survey", Signal Processing, vol. 91, No. 3, pp.386-426, March 2011.

[4] A. Kumar, R. Komaragiri, M. Kumar. "From pacemaker to wearable: techniques for ECG detection systems". J. Med. Syst. Vol. 42, №. 2, pp.1-34, February 2018.

[5] M.I. Skolnik, "Introduction to radar systems". McGraw-Hill. New York. 1980.

[6] C-W.Hsue, L-C. Tsai, Yi-H. Tsai“Time constant control of microwave integrators using transmission lines", IEEE Trans. Microwave ther. And techn. vol. 54, №.3, pp. 1006-1011, march 2006.

[7] H. Farid, E.P. Simoncelli, "Differentiation of discrete multidimensional signals", IEEE. Trans. image processing, vol. 13, No. 4, pp. 496-508, April. 2004.

[8] M.A.AL-Alaoui, "Novel FIR approximations of IIR differentiators with applcations to image edge detection", IEEE Proceedings of International Conference on Electronics, Circuits and Systems (ICECS), 11-14 Dec 2011, Beirut, Lebanon, 2011.

[9] B. Kumar, D.R. Choudhury, and Kumar A."On the design of linear phase FIR integrators for midband frequencies", IEEE Trans. Signal process. vol. 44, $\mathrm{N}^{\circ} .10, \mathrm{pp}$. 345-353, October.1996.

[10] B. Kumar, Kumar A.,"FIR linear phase approximations of frequency response $1 /(j \omega)$ for maximal flatness at an arbitrary frequency $\omega 0 \quad\left(\omega_{0}\left(0<\omega_{0}<\pi\right) ", \quad\right.$ IEEE Trans. Signal process. vol. $47, \mathrm{~N}^{\circ} .6$, pp. 1772-1775, June 1999.

[11] S. Singh, K. Singh, "Higher Order Low Pass FIR Digital Differentiators", International Journal of Computer Applications, Vol. 87, N.5, pp: $0975-8887$, February 2014.

[12] A. Aggarwal, M. Kumar, T.K.Rawat, "Design of Digital Differentiator Using the L1-Method and Swarm Intelligence-Based Optimization Algorithms", Arabian Journal for Science and Engineering, Vol. 44, pp.1917-1931, 2019.

[13] M. A. Al-Alaoui, "Al-Alaoui operator and the new transformation polynomials for discretization of analogue systems", Electr. eng. ,vol. 90, №. 6, pp. 455-467, january 2008.

[14] M. A. Al-Alaoui, "A class of second-order integrators and low-pass differentiators", IEEE. Trans. Circuits Syst. I Fundam. Theory Appl. vol .42, No. 4, pp. 220-223. April.1995.

[15] M. K. Jalloul, M. A. Al-Alaoui, "design of recursive digital integrators and differentiators using particle swarm optimization", International Journal of Circuit Theory and Applications, July 2015.

[16] N. Rawal, A. Bohra, "Design of Second Order Digital Integrator Using Simulation Annealing", Sch. J. Eng. Tech., vol.4, №.1, pp:89-94, January2016.

[17] S. K. Mitra, "Digital signal processing", 3nd edn. Mc-Graw Hill, 2006.

[18] V. Georgiev, "Some applied aspects of rational higher order S-Z transformations", radioengineering, Vol. $7, \mathrm{~N}^{\circ} .1$, pp: 19-26,April 1998.

[19] Y.Z. Povstenko, "Fundamental solutions to central symmetricproblems for fractional heat conduction equation and associated thermal stress", J. Therm.Stresses vol.31, pp:127148, February2008.

[20] M. Panitz, J. Paul, "A fractional boundary placement model using the transmission-line modeling (TLM) method", IEEE Trans. Microw. Theor. Tech., Vol. 57,pp:637-646, February2009.

[21] J.D. Gabano, T. Poinot, "Fractional modelling applied to heat conductivity and diffusivity estimation", Phys. Scripta, Vol. 136, pp. 16,October 2009.

[22] Y. Ferdi, "Computation of fractional order derivatives and integral via power series expansion and signal modeling", nonlinear dynamics. vol. 46, №.1-2, pp. 1-15, March. 2006.

[23] Y.Q.Chen, B.M. Vinagre, I. Podlubny, "Continued fraction expansion approaches to discretizing fractional order derivatives- an expository review", Nonlinear. Dyn, vol.38, №.1-4, pp.155-170, December 2004.

[24] C.C. Tseng, "Series expansion design of variable fractional order integrator and differentiator using logarithm", Signal 
processing, vol.88. №.9, pp. 2278-2292, September 2008.

[25] Y. Ferdi, "Impulse invariance- based method for the computation of fractional integral of order $0<\alpha<1$ ", Comput. andElectri. Eng. vol. 35, №. 5, pp. 722-729, March.2009.

[26] K. Steiglitz, L.E. Mc-Bride," A technique for the identification of linear systems", IEEE Trans. On Automat. Cont.vol. AC-10, №. 4, pp. 461-464, October.1965.

[27] C. Mekhnache, Y. Ferdi, "Using new hybrid Integrators to design IIR fractional order integrators via impulse invariance method", Cir. Syst. and signal processing, vol.33, Nº. 2, Springer, February2014.

[28] Ch. Mekhnache, N. Detouche, N. Balaska, "Design of New Variable Fractional Order IIR Graham-Lindquist/Euler Digital Differentiator",ICTAEE'18proceeding,10-11 Dec 2018, Skikda, Algeria, 2018.

[29] J. Le bihan, "Novel class of digital integrators and differentiators", llectro. Letter. vol. 29, №.11, pp. 971-973, May 1993.

[30] L. R. Rabiner, K. Steiglitz, "The design of wide-band recursive and nonrecursive digital differentiators", IEEE Trans. Audio Electroacoust.vol. 18, $\mathrm{N}^{\circ}$.2, pp. 204-209,June 1970.

[31] Ferdi Y. Boucheham B. "Recursive filter approximation of digital fractional differentiator and integrator based on Prony's method". in proc. ENSEIRB 1st IFAC Workshop Fractional different. Its Appl., Bordeaux, France.2004. 